Article

\title{
Postural Control in Workplace Safety: Role of Occupational Footwear and Workload
}

\author{
Harish Chander ${ }^{1, *}$, John C. Garner ${ }^{2}$, Chip Wade ${ }^{3}$ and Adam C. Knight ${ }^{1}$ \\ 1 Neuromechanics Laboratory, Department of Kinesiology, Mississippi State University, Mississippi State, \\ MS 39762, USA; aknight@colled.msstate.edu \\ 2 Department of Kinesiology and Health Promotion, Troy University, Troy, AL 36082, USA; jcgarner@troy.edu \\ 3 Department of Industrial and Systems Engineering, Auburn University, Auburn, AL 36849, USA; \\ lrw0002@auburn.edu \\ * Correspondence: hchander@colled.msstate.edu; Tel.: +1-(662)-202-7977
}

Academic Editor: Raphael Grzebieta

Received: 11 July 2017; Accepted: 27 July 2017; Published: 1 August 2017

\begin{abstract}
Maintaining postural stability is crucial, especially in hazardous occupational environments. The purpose of the study was to assess the role of three occupational footwear (low top shoe (LT); tactical work boot (TB) and steel-toed work boot (WB)) on postural stability when exposed to an occupational workload (4-h) involving standing/walking using the sensory organization test (SOT) equilibrium (EQ) scores and comparing current results with previously published postural sway variables from the same study. Fourteen male adults were tested on three separate days wearing a randomized occupational footwear, at the beginning (pre) and every $30 \mathrm{~min}$ of the 4-h workload until 240 th $\mathrm{min}$. SOT EQ scores were analyzed using a $3 \times 9$ repeated measures analysis of variance at an alpha level of 0.05 . Significant differences between footwear was found in eyes open $(p=0.03)$ and eyes closed $(p=0.001)$ conditions. Pairwise comparisons revealed that LT had significantly lower postural stability compared to TB and WB. No other significant differences were found between footwear and over time. Significant differences between footwear can be attributed to design characteristics of footwear. Lack of significant differences over time suggests that, even though the average EQ scores decreased during the workload implying less postural stability, SOT EQ scores alone may not be sufficient to detect postural stability changes over the 4 -h workload.
\end{abstract}

Keywords: postural stability; balance; occupational footwear; workload; sensory organization test (SOT)

\section{Introduction}

Postural control is regarded as a skill where the central nervous system utilizes sensory and motor systems to maintain postural stability [1]. Degradation or defect in any of these systems increases the probability of postural instability and hence a possibility of a fall [2]. Postural instability is a hazardous situation for employees at a workplace, especially in the heavy engineering, manufacturing and construction sectors. The physical exertion due to such occupations and the environmental constraints of the workplace, create greater demands on the postural control system and increase the risk of occupational falls [3]. Previous studies have identified postural instability when exposed to an occupational workload or physical exertion [4-6]. Increases in postural sway, which often accompany fatigue, mark decreased stability. These increases in sway can be attributed to impairments of any of these systems [2]. Standing and walking for long periods of work hours have been reported to cause postural instability on flat surfaces [5,7] and on inclined or unstable surfaces [8,9]. Muscular fatigue or exertion, resulting from such physical demands of the occupation is considered as an important intrinsic human factor that plays a significant role in postural control. When muscular fatigue ensues, 
the ability of the postural system to handle disturbances is inhibited [10] which can be quantified by increased postural sway indicating decreased postural stability. The onset of muscular fatigue, thus, requires more exertion from the postural control system in order to maintain correct posture [11]. One extrinsic or non-human factor that can impact postural control is the type of footwear worn, which forms the contacting medium between the body and the standing/walking environment. They also play a significant role in relaying afferent somatosensory and proprioceptive sensory information from the environment and the feet, to the postural control system, aiding in maintaining postural stability. Previous studies have reported the impact of different footwear types, identifying both advantageous and disadvantageous effects in maintaining postural stability that were attributed to specific design characteristics, such as heel height, mass, boot shaft height and flexibility, mid and outer-sole thickness, slip-resistant outsoles, lacing types and material of the footwear [4,5,12-16].

More recently, the impact of three occupational footwear and an occupational workload on postural stability have been analyzed [5]. The findings from this study report postural stability changes using the sensory organization test (SOT) on the Neurocom Equitest ${ }^{\mathrm{TM}}$ (Neurocom International Inc, Clackamas, OR, USA), quantified by anterior-posterior and medial-lateral sway velocities and root mean square sway derived from center of pressure (COP) measures. The SOT was administered at 9-time points in 30-min increments over the 4-h walking workload with three different occupational footwear (low-top shoe, high-top tactical work boot and high-top steel toed work boot). It was identified that postural stability decreased over time due to the workload and that high-top boots provided greater postural stability compared to the low-top shoe [5]. Postural sway variables derived from COP excursions have been used to quantify balance performance $[4,5,15,16]$. Similarly, the equilibrium scores from the SOT have been used widely for postural stability assessments in a variety of populations for its ease of access and immediate availability of results [15,17-19]. However, these postural stability assessment scores may not be sufficient to identify all existing differences. Although, previous studies have identified the influence of footwear and different types of workload on postural stability, there is still a need for assessing the interaction of different occupational footwear types and occupational workloads on postural stability, specifically using the equilibrium scores from the SOT. Therefore, the purpose of this paper was to examine whether differences in postural stability exist while wearing three different types of occupational footwear, when exposed to a simulated extended duration occupational workload involving prolonged standing and walking and to compare these findings with the postural sway variables that have been previously reported from the study [5]. It was hypothesized that postural stability will be altered based on the type of occupational footwear and when exposed to a simulated occupational workload involving prolonged duration standing and walking.

\section{Materials and Methods}

Fourteen healthy male adults (age: $23.6 \pm 1.2$ years; height: $181 \pm 5.3 \mathrm{~cm}$; mass: $89.2 \pm 14.6 \mathrm{~kg}$ ), participated in this study. Sample size was estimated using G-Power software with a desired power of 0.8 , effect size of 0.25 and alpha level of 0.05 and based on previous studies from the laboratory. Sixteen participants were recruited and two dropped out from the study. Only males were selected for the study as the construction and manufacturing sector has a predominant male population as employees and due to the availability of only male size shoes. The study followed a repeated measures design with participants tested on three separate days wearing a randomly assigned occupational footwear (low top shoe (LT) (mass: $0.39 \pm 0.06 \mathrm{~kg}$; boot shaft height: $9.5 \mathrm{~cm}$; heel height: $2.1 \mathrm{~cm}$ ); Tactical Work Boot (TB) (mass: $0.53 \pm 0.08 \mathrm{~kg}$; boot shaft height: $16.5 \mathrm{~cm}$; heel height: $3.3 \mathrm{~cm}$ ) and Steel-Toed Work Boot (WB) (mass: $0.89 \pm 0.05 \mathrm{~kg}$; boot shaft height: $18.5 \mathrm{~cm}$; heel height: $3.8 \mathrm{~cm}$ )) (Figure 1), separated by a minimum of $72 \mathrm{~h}$. All participants were asked for their respective shoes sizes. The only instruction provided to them were, to make sure the fit of the shoe was not too tight or too loose to wear and to lace the shoes normally. All participants read and signed the informed consent approved by the University's Institutional Review Board (IRB) (IRB\# 11-150) and were exposed to a 
familiarization session involving all balance test conditions. The experimental session started with a 5 min basic warm-up exercises such as walks, jogs and high knees. To simulate an occupational workload that involved extended durations of standing and walking, participants were instructed to walk with a self-selected pace and a self-selected path $[6,8]$ on a $9 \mathrm{~m} \times 6 \mathrm{~m}$, flat vinyl tiled surface for the entire testing time of $4 \mathrm{~h}$, until every 30-min interval (Pre, $30 \mathrm{~min}, 60 \mathrm{~min}, 90 \mathrm{~min}, 120 \mathrm{~min}, 150 \mathrm{~min}$, $180 \mathrm{~min}, 210 \mathrm{~min}$ and $240 \mathrm{~min}$ ) to complete the postural stability assessment. During the 4-h walking protocol, participants were allowed to stand stationary for a few seconds to a couple of minutes but were not allowed to sit down or rest.

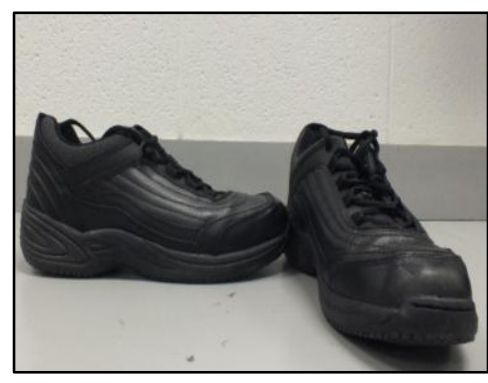

Low Top Shoe (LT)

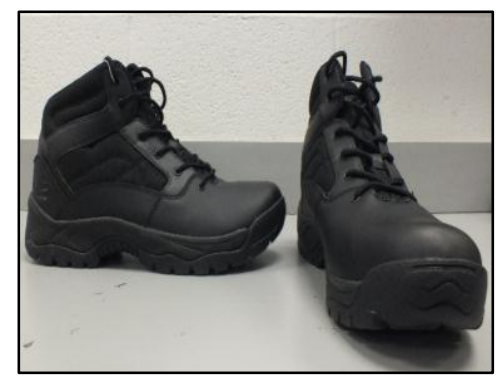

Tactical Work Boot (TB)

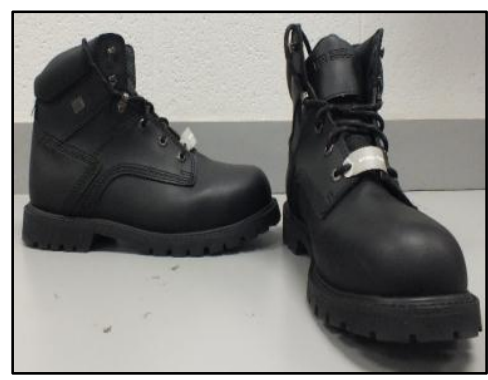

Steel Toed Work Boot (WB)

Figure 1. Occupational footwear: Low-top shoe (LT); tactical work boot (TB); and steel-toed work boot (WB).

The SOT on the Neurocom Equitest ${ }^{\mathrm{TM}}$ was used to assess postural stability that consisted of a total of six testing conditions (eyes open-EO, eyes closed-EC, Eyes Open Sway Referenced Vision-EOSRV, Eyes Open Sway Referenced Platform-EOSRP, Eyes Closed Sway Referenced Platform-ECSRP and Eyes Open Sway Referenced Vison \& Platform-EOSRVP). Each condition consisted of 3 trials of $20 \mathrm{~s}$ balance tests. Equilibrium (EQ) score which is an overall representation of the postural stability during the SOT was used as a postural stability measure, where higher EQ scores represent less sway therefore better postural stability. The composite score (COMP) is a cumulative score of all six conditions and similarly, higher COMP EQ scores represent better postural stability. SOT EQ scores were evaluated using a $3 \times 9$ (Footwear [LT, TB, WB]) $\times$ (Time [Pre, 30, 60, 90, 120, 150, 180, 210 and 240]) within subjects repeated measures analysis of variance (repeated measures ANOVA), independently for the six SOT balance conditions (EO, EC, EOSRV, EOSRP, ECSRP and EOSRVP) and individually for the COMP EQ score to identify any existing differences between the footwear types and across the exposure time. If footwear $\times$ time interaction or footwear and time main effect significance was found, it was followed with post-hoc simple effects tests or post-hoc pairwise comparisons with a Bonferroni correction, respectively. All statistical analyses were performed using SPSS statistical software at an alpha level of 0.05 .

\section{Results}

The repeated measures ANOVA revealed significant main effect differences for footwear in the EO testing condition $\left(\mathrm{F}(2,26)=4.020, p=0.03, \eta_{p}^{2}=0.236\right)$ (Figure 2$)$ and in the EC testing condition $\left(\mathrm{F}(2,26)=9.492, p=0.001, \eta_{p}{ }^{2}=0.422\right)$ (Figure 3). Pairwise comparisons using a Bonferroni correction further revealed that TB and WB had significantly higher EQ scores compared to the LT in the EC condition and TB had significantly higher EQ scores than LT in the EO condition. No other significant differences were seen with the EQ scores across all SOT balance testing conditions including the COMP EQ score. 


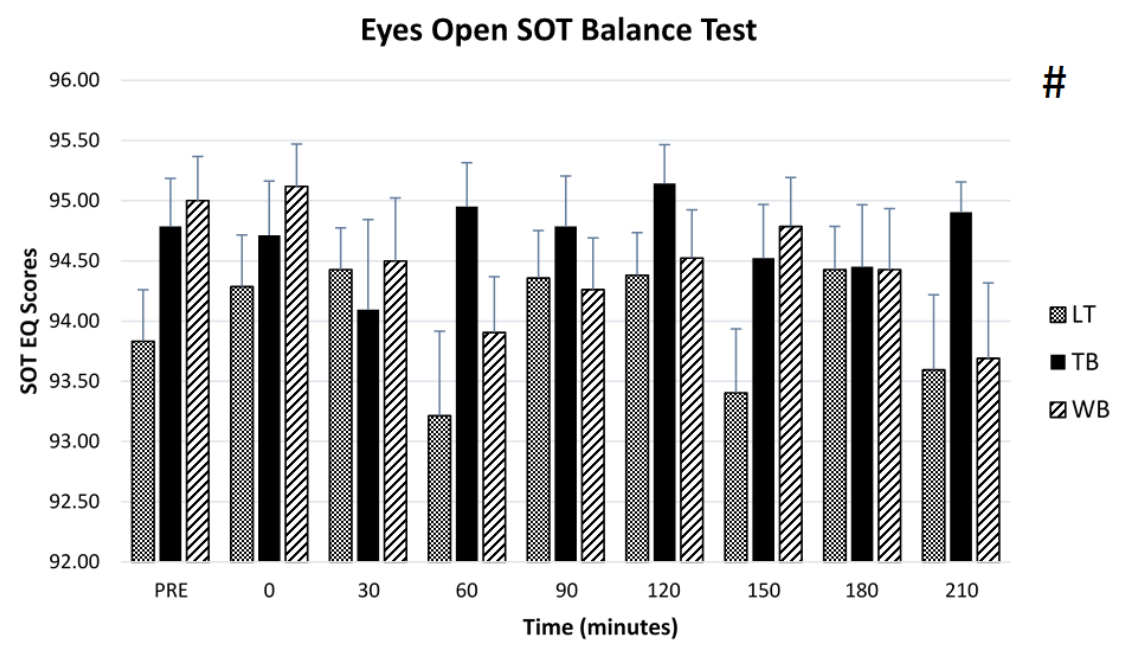

Figure 2. Mean Sensory Organization Test (SOT) Equilibrium (EQ) scores from the Eyes Open (EO) condition for all three footwear (LT, TB and WB) at nine time points. \# represents a significant main effect for footwear. Bars represent standard errors.

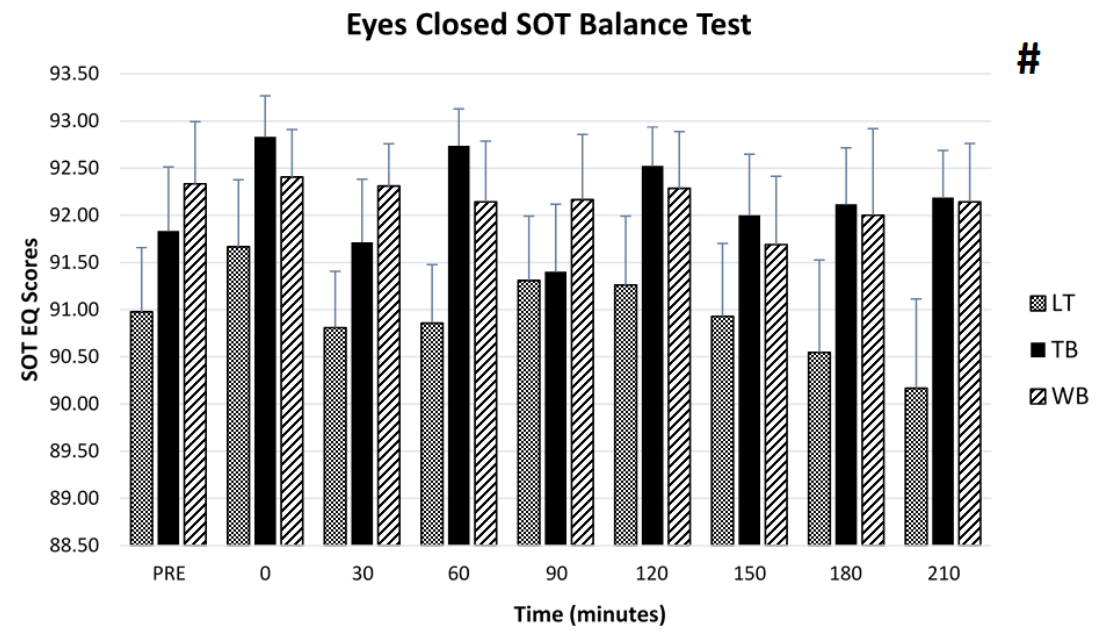

Figure 3. Mean SOT EQ scores from the eyes closed (EC) condition for all three footwear (LT, TB and $\mathrm{WB}$ ) at nine time points. \# represents a significant main effect for footwear. Bars represent standard errors.

\section{Discussion}

The purpose of the study was to examine the impact of three occupational footwear and a simulated occupational workload on postural stability and to identify if differences existed in SOT EQ scores in comparison to postural sway variables derived from COP excursions reported in previously published data from the study [5]. The findings of this study suggest that there were significant differences in postural stability among the occupational footwear types with the LT eliciting significantly lower equilibrium scores compared to the TB and WB in both $\mathrm{EO}$ and $\mathrm{EC}$ testing conditions. The significant footwear differences that existed in this analysis supported the COP postural sway variables previously reported from the study [5]. The lowered postural stability can be attributed to the design characteristics of these footwear. Although, the results align with previously reported data from the study, significant differences in SOT EQ scores were only found for the EO and EC conditions compared to the significant differences in COP postural sway variables that existed in EO, EC, EOSRV and EOSRP conditions. No significant differences were seen with time as a main effect, indicating that the extended duration of standing and walking did not influence SOT EQ scores, regardless of 
the footwear used. However, this finding is contrary to the COP postural sway variables previously reported from this study [5], where significant differences over time were reported, with balance decrements during the $4 \mathrm{~h}$ of standing/walking. The lowered postural stability was attributed to the physical workload placing greater demands on the postural control system [5].

Footwear design characteristics that have been shown to aid in postural stability include a high boot shaft [20,21], lower mass [4], low heel height [14,15] and firm-hard midsoles [14,22]. The lowered balance performance in the LT may be attributed to the below ankle boot shaft height. Previous literature has supported that an elevated boot shaft that extends above the ankle joint offers greater balance performance by providing support around the ankle joint axis and increasing somatosensory feedback for improved joint position sense $[4,5,14,16,21]$. The TB and WB with boot shaft heights of $16.5 \mathrm{~cm}$ and $18.5 \mathrm{~cm}$ respectively provides the ankle joint with support and stability to minimize postural sway in comparison to the shaft height of $9.5 \mathrm{~cm}$ in the LT. The use of LT resulted in a relatively greater balance decrement, especially when vision was absent and when the postural control system is relying more on the somatosensory input for postural stability maintenance. An increase in the mass of the boot has been shown to cause an increase in energy expenditure by $0.7 \%-1.0 \%$ of locomotion for each $100 \mathrm{~g}$ increase in the weight of the footwear [23-25] and thereby a faster rate of muscular fatigue which may lead to decrements in balance. The WB and TB despite having a greater mass, had superior balance performance, which can be related to their elevated boot shaft height aiding in better postural stability. Moreover, insole and midsole type and thickness have also been shown to impact postural stability, where softer material have been shown to be detrimental to balance performance $[14,22]$. As such, the LT had a softer insole and midsole compared to the TB and WB, which might have contributed to the inferior postural stability. Finally, increased heel height has been associated with decreased stability [14] by shifting the total body center of mass more anteriorly in standing and placing the body in more unstable position. However, the LT had the lowest heel height, $2.1 \mathrm{~cm}$ compared to $3.5 \mathrm{~cm}$ and $3.8 \mathrm{~cm}$ for the TB and WB respectively, indicating greater postural stability with the use of the high-top boots (TB and WB). Thus, the boot shaft heights appeared to play a greater role for maintaining postural stability in comparison to heel height or mass of the footwear.

It is known that the postural control system is placed on greater demands with muscular fatigue as a result of a physiological workload leading to postural instability $[2,10,11]$. Postural instability due to muscular exertion and fatigue are usually reported for workloads that are greater than $50 \%$ of maximal voluntary contraction (MVC) and greater than 33\% of maximal aerobic capacity (MAC) [26]. However, workloads experienced in this study resemble an occupational setting that are less than $15 \%$ of MVC and less than 33\% of MAC [26]. Although, the mean SOT EQ scores lowered as the extended duration workload continued, a significant time main effect was not seen in this analysis, implying that the SOT EQ scores were not influenced by the workload. However, postural sway variables such as sway velocities and root mean square sway in the anterior-posterior and medial-lateral directions reported previously from this study [5], demonstrate a significant time effect, suggesting that the workload that included a self-selected pace and path walking for 4 hours caused balance decrements over time [5]. Hence, the EQ scores provided by the NeuroCom Equitest ${ }^{\mathrm{TM}}$ may not be sufficient to have a full understanding of the postural changes and be adequate to identify balance differences that are evident from an extended duration workload, especially in healthy individuals. The need for more complex postural control analyses, rather than relying on the SOT EQ scores has been previously suggested $[27,28]$ and the results from the current study support this notion. The direct balance or postural stability scores provided by the testing equipment are of great value, for its ease of access and immediate availability. Moreover, the SOT EQ scores have been previously used with great success in many studies assessing a variety of population such elderly [29] and pathological or clinical populations [30,31]. However, the SOT EQ scores from the current study assessing healthy populations did not indicate significant differences over time and indicated significant differences in footwear only in the $\mathrm{EO}$ and $\mathrm{EC}$ conditions. The COP excursion analysis using postural sway variables from the same study reported significant differences in footwear, over time and also in the EO, EC, EOSRV 
and EOSRP conditions [5], supporting previous literature for the need for more detailed postural sway analyses in place of the SOT EQ score. More importantly, it has also been shown that repeated assessments of postural stability using the SOT and its analysis of the EQ score, on healthy young populations can induce a learning effect especially in the EOSRP, ECSRP and EOSRVP conditions [32]. Thus, further analysis using COP excursions calculating postural sway velocities, postural sway root mean square, sway area, postural stability index, $95 \%$ ellipsoid area etc. is crucial especially when testing healthy populations.

There are a few limitations of the study that need to be considered before generalizing the results from the current study. Only healthy male populations were analyzed for this study, as a predominant number of construction and manufacturing workers are males, and due to the availability of male size footwear. Moreover, the sample consisted of healthy young adults, whose postural control is superior compared to aged individuals. However, with the use of a repeated measures design, the impact of footwear types on postural stability were assessed, independent of age. Future studies should focus on different footwear types and different types of occupational workloads to gain a better understanding of the role of such factors on the postural control system.

\section{Conclusions}

Occupational footwear and workload play a critical role in influencing postural stability. Occupational footwear which brings together the best design characteristics including an elevated boot shaft, lower mass with steel toe protection and moderate heel height would be more suitable for better postural stability in an occupational setting. The EQ scores exhibited by the LT was significantly lower in comparison to the tactical and work boots, suggesting it to be the footwear type that provides the least assistance in aiding postural stability. The results from the current study identifies that the LT exhibits increased postural sway and subsequently demonstrated the lowest postural stability [3-5] during simulated occupational workloads. Hence the use of LT has a greater potential to result in falls and fall related injuries at the workplace. Moreover, the SOT EQ scores may not be sufficient for identifying postural stability differences over time and may require further postural sway analysis using COP excursions.

Acknowledgments: This project was not supported by any external grants.

Author Contributions: Harish Chander, John C. Garner and Chip Wade conceived and designed the experiments; Harish Chander performed the experiments; Harish Chander and Adam C. Knight analyzed the data; Harish Chander, John C. Garner, Chip Wade and Adam C. Knight contributed reagents/materials/analysis tools; Harish Chander wrote the paper.

Conflicts of Interest: The authors declare no conflict of interest.

\section{References}

1. Winter, D.A. Human balance and postural control in standing and walking. Gait Posture 1995, 3, 193-214. [CrossRef]

2. Lepers, R.; Bigard, A.X.; Diard, J.P.; Gouteyron, J.F.; Guezennec, C.Y. Posture control after prolonged exercise. Eur. J. Appl. Physiol. Occup. Physiol. 1997, 76, 55-61. [CrossRef] [PubMed]

3. Kincl, L.D.; Bhattacharya, A.; Succop, P.A.; Clark, C.S. Postural sway measurements: A potential safety monitoring technique for workers wearing personal protective equipment. Appl. Occup. Environ. Hyg. 2002, 17, 256-266. [CrossRef] [PubMed]

4. Garner, J.C.; Wade, C.; Garten, R.; Chander, H.; Acevedo, E. The influence of firefighter boot type on balance. Int. J. Ind. Ergon. 2013, 43, 77-81. [CrossRef]

5. Chander, H.; Garner, J.C.; Wade, C. Impact on balance while walking in occupational footwear. Footwear Sci. 2014, 6, 59-66. [CrossRef]

6. Wade, C.; Garner, J.C.; Redfern, M.S.; Andres, R.O. Walking on ballast impacts balance. Ergonomics 2014, 57, 66-73. [CrossRef] [PubMed] 
7. Cham, R.; Redfern, M.S. Effect of flooring on standing comfort and fatigue. Hum. Factors 2001, 43, 381-391. [CrossRef] [PubMed]

8. Wade, C.; Davis, J. Postural sway following prolonged exposure to an inclined surface. Saf. Sci. 2009, 47, 652-658. [CrossRef]

9. Wade, C.; Davis, J.; Weimar, W.H. Balance and exposure to an elevated sloped surface. Gait Posture 2014, 39, 599-605. [CrossRef] [PubMed]

10. Yaggie, J.A.; McGregor, S.J. Effects of isokinetic ankle fatigue on the maintenance of balance and postural limits. Arch. Phys. Med. Rehabil. 2002, 83, 224-228. [CrossRef] [PubMed]

11. Corbeil, P.; Blouin, J.; Begin, F.; Nougier, V.; Teasdale, N. Perturbation of the postural control system induced by muscular fatigue. Gait Posture 2003, 18, 92-100. [CrossRef]

12. Hosoda, M.; Yoshimura, O.; Takayanagi, K.; Kobayashi, R.; Minematsu, A.; Sasaki, H.; Maejima, H.; Matsuda, Y.; Araki, S.; Nakayama, A.; et al. The effect of footwear on standing posture control. J. Phys. Ther. Sci. 1998, 10, 47-51. [CrossRef]

13. Maki, B.; Perry, S.; Scovil, C.; Peters, A.; McKay, S.; Lee, T.; Corbeil, P.; Fernie, G.; McIlroy, W. Interventions to Promote More Effective Balance-Recovery Reactions in Industrial Settings: New Perspectives on Footwear and Handrails. Ind. Health 2008, 46, 40-45. [CrossRef] [PubMed]

14. Menant, J.; Steele, J.; Menz, H.; Munro, B.; Lord, S. Effects of Footwear Features on Balance and Stepping in Older People. Gerontology 2008, 54, 18-23. [CrossRef] [PubMed]

15. Chander, H.; Morris, C.E.; Wilson, S.J.; Garner, J.C.; Wade, C. Impact of alternative footwear on human balance. Footwear Sci. 2016, 8, 165-174. [CrossRef]

16. Chander, H.; Wade, C.; Garner, J.C. Impact of Occupational Footwear on Dynamic Balance Perturbations. Footwear Sci. 2015, 7, 115-126. [CrossRef]

17. Nashner, L. Computerized Dynamic Posturography. In The Handbook of Balance Function and Testing; Jacobson, G., Newman, C., Kartush, J., Eds.; Mosby Year Book: St. Louis, MO, USA, 1993; pp. 261-279.

18. Guskiewicz, K.M.; Perrin, D.H. Research and clinical applications of assessing balance. J. Sport Rehabil. 1996, 5, 45-63. [CrossRef]

19. Chander, H.; Dabbs, N.C. Balance Performance and Training among Female Athletes. Strength Cond. J. 2016, 38, 8-13. [CrossRef]

20. Bohm, H.; Hosl, M. Effect of boot shaft stiffness on stability joint energy and muscular co-contraction during walking on uneven surface. J. Biomech. 2010, 43, 2467-2472. [CrossRef] [PubMed]

21. Cikajlo, I.; Matjačić, Z. The influence of boot stiffness on gait kinematics and kinetics during stance phase. Ergonomics 2007, 50, 2171-2182. [CrossRef] [PubMed]

22. Hijmans, J.; Geertzen, J.; Dijkstra, P.; Postema, K. A systematic review of the effects of shoes and other ankle or foot appliances on balance in older people and people with peripheral nervous system disorders. Gait Posture 2007, 25, 316-323. [CrossRef] [PubMed]

23. Franz, J.R.; Wierzbinski, C.M.; Kram, R. Metabolic cost of running barefoot versus shod: Is lighter better. Med. Sci. Sports Exerc. 2012, 44, 1519-1525. [CrossRef] [PubMed]

24. Frederick, E.C.; Daniels, J.T.; Hayes, J.W. The effect of shoe weight on the aerobic demands of running. In Current Topics in Sports Medicine, Vienna (Austria); Bachl, N., Prokop, L., Suckert, R., Eds.; Urban \& Schwarzenberg: Vienna, Austria, 1984; pp. 616-625.

25. Jones, B.H.; Toner, M.M.; Daniels, W.L.; Knapik, J.J. The energy cost and heart-rate response of trained and untrained subjects walking and running in shoes and boots. Ergonomics 1984, 27, 895-902. [CrossRef] [PubMed]

26. Davidson, B.S.; Madigan, M.L.; Nussbaum, M.A. Effects of lumbar extensor fatigue and fatigue rate on the postural sway. Eur. J. Appl. Phys. 2004, 93, 183-189. [CrossRef] [PubMed]

27. Chaudhry, H.; Findley, T.; Quigley, K. S.; Ji, Z. Postural stability index is a more valid measure of stability than equilibrium score. J. Rehabil. Res. Dev. 2005, 42, 547-556. [CrossRef] [PubMed]

28. Chaudhry, H.; Findley, T.; Quigley, K.S.; Bukiet, B. Measures of postural stability. J. Rehabil. Res. Dev. 2004, 41, 713-720. [CrossRef] [PubMed]

29. Cohen, H.; Heaton, L.G.; Congdon, S.L.; Jenkins, H.A. Changes in sensory organization test scores with age. Age Ageing 1996, 25, 39-44. [CrossRef] [PubMed]

30. Nocera, J.; Horvat, M.; Ray, C.T. Effects of home-based exercise on postural control and sensory organization in individuals with Parkinson disease. Parkinsonism Relat. Disord. 2009, 15, 742-745. [CrossRef] [PubMed] 
31. Riemann, B.L.; Guskiewicz, K.M. Effects of mild head injury on postural stability as measured through clinical balance testing. J. Athl. Train. 2000, 35, 19-25. [PubMed]

32. Wrisley, D.M.; Stephens, M.J.; Mosley, S.; Wojnowski, A.; Duffy, J.; Burkard, R. Learning effects of repetitive administrations of the sensory organization test in healthy young adults. Arch. Phys. Med. Rehabil. 2007, 88, 1049-1054. [CrossRef] [PubMed] 